\title{
BEST POSSIBLE BOUNDS FOR ORDERED POSITIVE NUMBERS USING THEIR SUM AND PRODUCT
}

\author{
JORMA KAARLO MERIKOSKI AND ARI VIRTANEN
}

\begin{abstract}
Best possible bounds for real numbers $\lambda_{1} \geqslant \cdots \geqslant \lambda_{n}>0$ with prescribed sum $a=\lambda_{1}+\cdots+\lambda_{n}$ and product $d=\lambda_{1} \cdots \lambda_{n}$ are presented. These bounds can be expressed algebraically only in certain special cases. In the general case, explicit bounds are found by using extra bounds. The results are applied to eigenvalue estimation, when the $\lambda_{k}$ 's are regarded as eigenvalues of an $n$ by $n$ matrix $A, a=\operatorname{tr} A$, and $d=\operatorname{det} A$. The case when the eigenvalues are real but not necessarily positive is also discussed. The bounds are compared with bounds using $a$ and $b=\lambda_{1}^{2}+\cdots+\lambda_{n}^{2}$; i.e, with eigenvalue bounds using $\operatorname{tr} A$ and $\operatorname{tr} A^{2}$.
\end{abstract}

Mathematics subject classification (2000): 26E60, 26D15, 15A18.

Key words and phrases: Inequality, sum, product, mean, eigenvalue, trace, determinant.

\section{REFERENCES}

[1] B. Grone, C. Johnson, E. M. De SÁ, And H. Wolkowicz, Improving Hadamard's Inequality, Linear and Multilienear Algebra 16(1984), 305-322.

[2] H. W. Guggenheimer, A. S. Edelman, And C. R. Johnson, A simple estimate of the condition number of a linear system, College Math. J., 26(1995), 2-5.

[3] S. T. JENSEN, The Laguerre-Samuelson inequality with extensions and applications in statistics and matrix theory, Master's thesis, Department of Mathematics and Statistics, McGill University, 1999.

[4] S. T. JENSEN AND G. P. H. STYAN, Some comments and a bibliography on the Laguerre-Samuelson inequality with extensions and applications in statistics and matrix theory, in $\mathrm{H}$. M. Srivastva and T. M. Rassias (eds.) Analytic and Geometric Inequalities and Their Applications, Kluwer, 2000.

[5] J. K. MERIKOSKI, H. SARRIA, AND P. TARAZAGA, Bounds for singular values using traces, Linear Algebra Appl., 210(1994), 227-254.

[6] J. K. Merikoski, G. P. H. Styan, AND H. Wolkowicz, Bounds for ratios of eigenvalues using traces, Linear Algebra Appl. 55(1983), 105-124.

[7] J. K. Merikoski, U. URPala, AND A. VirTanen, Upper bounds for the ratio of the largest and smallest eigenvalues, Department of Mathematical Sciences, University of Tampere, Report A 307, 1996.

[8] J. K. Merikoski, U. Urpala, A. Virtanen, T.-Y. TAm, And F. Uhlig, A best upper bound for the 2-condition number of a matrix, Linear Algebra Appl. 254(1997), 355-365.

[9] J. K. Merikoski and A. Virtanen, Bounds for eigenvalues using the trace and determinant, Department of Mathematical Sciences, University of Tampere, Report A 308, 1996.

[10] J. K. MERIKOSKI AND A. ViRTANEN, Bounds for eigenvalues using the trace and determinant, Linear Algebra Appl., 264(1997), 101-108.

[11] J. K. Merikoski, A. VirTanen, AND O. PikhURKo, Majorization bounds for vectors and bounds for sums using a sum constraint and a convex constraint, Manuscript.

[12] J. K. MERIKOSKI AND H. WOLKOWICZ, Improving eigenvalue bounds using extra bounds, Linear Algebra Appl., 68(1985), 93-113.

[13] H. WOLKOWICZ AND G. P. H. STYAN, Bounds for eigenvalues using traces, Linear Algebra Appl., 29(1980), 471-506. 